\title{
A Simulation of Focal Brain Stimulation using Metamaterial Lenses
}

\author{
Luis Gomez*, Luis Hernandez, Anthony Grbic, Eric Michielssen \\ Radiation Laboratory, Department of Electrical and Computer Engineering, \\ University of Michigan, Ann Arbor, MI \\ Email: luisgo@umich.edu
}

\section{Introduction}

Transcranial magnetic stimulation (TMS) is a relatively recent technique for studying brain function and treating neurological disorders [1]. In TMS, one or more coils carrying time varying currents located near the scalp generate magnetic fields inside the brain that in turn induce electric fields and eddy-currents in the conductive brain tissue (Fig.1). Whenever a nerve fiber is aligned with the induced electric field, a current is produced in the axon, which in turn depolarizes its membrane [2]. Unfortunately, standard TMS coils stimulate large lateral regions of tissue near the scalp. To broaden the scope of current TMS applications, new applicators capable of delivering focused magnetic fields deep into the brain are called for [3].

Recently, it was shown that negative refractive index metamaterials (MM) can be used to focus magnetic fields in the near-field [4]. We have implemented a quasi-static finitedifference code [5] to simulate eddy-currents inside the conductive brain when excited by magnetic fields focused through a MM lens. Initial results show that coils used in conjunction with MM lenses produce more compact brain excitations than lens-free setups.

\section{Basic Guidelines}

Finite difference scheme. Our code is not unlike that proposed by Cerri et al. [5] though modified to allow for the presence of MM lenses. For the range of frequencies used in TMS, displacements currents can be neglected as they are much smaller than conduction currents. The same holds true for magnetic fields scattered by the brain as they are small relative to incident magnetic fields. We solve eqns. 1-4 below using a two step process (Figs. 2a-b). First, known driving coil currents are used to calculate the magnetic field in the presence of a MM lens by enforcing eqns. 1 and 2 on a cubic lattice. Second, previously obtained magnetic fields are used to calculate electric fields and eddy currents in brain tissue using an MRI derived conductivity map by enforcing eqns. 3 and 4 .

$$
\begin{gathered}
\oint_{C} \mathbf{H}(\mathbf{r}) \cdot d \mathbf{l}=\iint_{S} \mathbf{J}^{s}(\mathbf{r}) \cdot d \mathbf{s}, \\
\iint_{S_{c}} \mu(\mathbf{r}) \mathbf{H}(\mathbf{r}) \cdot d \mathbf{s}=0, \\
\oint_{C} \mathbf{E}(\mathbf{r}) \cdot d \mathbf{l}=-\frac{d}{d t} \iint_{S} \mu(\mathbf{r}) \mathbf{H}(\mathbf{r}) \cdot d \mathbf{s}, \\
\iint_{S_{c}} \sigma(\mathbf{r}) \mathbf{E}(\mathbf{r}) \cdot d \mathbf{s}=0 .
\end{gathered}
$$

In the above equations, $\mathbf{J}^{s}(\mathbf{r})$ is the coil current, $\mathbf{E}(\mathbf{r})$ is the total electric field, $\mathbf{H}(\mathbf{r})$ is the total magnetic field produced by the TMS coil in the presence of the MM lens, and $\mu(\mathbf{r})$ 
and $\sigma(\mathbf{r})$ are the permeability and conductivity at $\mathbf{r}$. The time derivative in eqn.3 is assumed to equate to a linear scalar factor multiplying the magnetic field derived from the time derivative of the coil current. In eqns. 1 and $3, \mathrm{C}$ is an arbitrary contour enclosing a surface $\mathrm{S}$, and in eqns. 2 and $4, S_{c}$ is an arbitrary closed surface.

Characterization measures. The effects of the induced fields on the brain are complex, and highly dependent on the magnitude and timing of the TMS pulse [6]. Although small fields can potentially cause neurons to depolarize, for most scenarios of interest neuronal activity only occurs when the electric field magnitude exceeds $150 \mathrm{~V} / \mathrm{m}$. As typically the intensity of the electric field drops rapidly as it penetrates the brain, only neurons near the top of the brain are activated. Increasing the amplitude of the coil currents of course deepens the region with above-threshold electric fields, but also widens its lateral cross section. In other words, an increase in the penetration depth (the distance from the top of the brain to the lowest point with fields in excess of $150 \mathrm{~V} / \mathrm{m}$ (Fig. 2c)) typically is associated with an increase in the excitation volume (the brain volume with fields in excess of $150 \mathrm{~V} / \mathrm{m}$ (Fig 2c)). Our goal is to design TMS systems that produce sharp excitations in the brain, maximizing the excitation volume for a given penetration volume and study Pareto trade-offs between both objectives.

\section{Results}

Effect of MM lens. To study the effect of a MM near-field lens on a typical TMS setup, we performed two simulations. In the first simulation a 'figure-8' coil resides symmetrically relative to the head's "symmetry plane" (Fig. 3a), $8 \mathrm{~mm}$ above the brain; no MM lens is present. In the second simulation the same figure- 8 coil resides $14 \mathrm{~mm}$ above a MM lens that has been shown by Pendry [3] to produce a perfect image $14 \mathrm{~mm}$ beneath the lower lens surface. Because this surface resides $8 \mathrm{~mm}$ above the brain (Fig. $2 \mathrm{~b}$ ), this setup is expected to produce an image of the figure- 8 coil $6 \mathrm{~mm}$ into the brain. The MRI brain image is comprised of $80 \times 87 \times 44$ cubic cells with voxel dimensions of $2 \mathrm{~mm} \times 2 \mathrm{~mm} \times 2 \mathrm{~mm}$. The time derivative of the coil current is $108 \mathrm{~A} / \mu \mathrm{s}$ and the individual coil radius is $23 \mathrm{~mm}$. The MM lens has dimensions $460 \mathrm{~mm} \times 230 \mathrm{~mm} \times 28 \mathrm{~mm}$. Figure 4 shows electric field magnitudes across a transverse slice $6 \mathrm{~mm}$ into the brain, as well as coronal and sagittal slices through the center of the brain. The incident field of the MM setup is much more compact at this thickness. Correspondingly, in the presence of a brain the MM setup produces a more compact spot size. However, the sagittal and coronal planes show that the MM setup does not produce excitations that penetrate as deeply into the brain as the setup without a MM lens. These results clearly demonstrate a depth to volume tradeoff when using MM TMS setups.

Tradeoff study. To further study this depth to volume tradeoff we studied the effect of the MM lens shape, thickness, coil current intensity, and placement of the head relative to the MM lens and coil. For these simulations we used a three-sphere model of the head [3] and a 'figure-8' coil (Fig. 5). Figure 6 shows Pareto fronts that illustrate optimal penetration depth to volume trade-offs derived by varying the above parameters. We found that the use of a MM lens always results in smaller excitation volume for a given penetration depth. This means that the TMS setups leveraging MM lenses perform superior to classical setups. More research is needed to determine how MM lenses enhance TMS setups using 'Hesed' coils [3] and TMS setups leveraging multiple small coils. 


\section{References}

[1] George M.; Nahas Z.; Kozel A.; Li X.; Yamanaka K.; Mishori A.; Bohning D. ," Mechanisms and the Current State of Transcranial Magnetic Stimulation," CNS Spectrums , vol.8, no.7, pp. 496-514. Jul. 2003

[2] Ilmoniemi, R. J.; Virtanen, J.; Ruohonen, J.; Karhu, J.; Aronen, H. J.; Bohning D.; Naatanen, R., " Neuronal responses to magnetic stimulation reveal cortical reactivity and connectivity," Neuroreport, vol.8, no.16, pp. 3537-3540. Nov. 1997

[3] Roth Y.; Zangen A.; J.; Hallett M., "A coil design for transcranial magnetic stimulation of deep brain regions," J Clin Neurophysiol, vol.19, no.4, pp. 361-370. Aug. 2002

[4] Pendry, J. B., "Negative refraction makes a perfect lens," Phys. Rev. Lett., vol.85, no.18, pp. 3967-3969. April. 2000

[5] Cerri, G.; De Leo, R.; Moglie, F.; Schiavoni, A., "An accurate 3-D model for magnetic stimulation of the brain cortex," J Med Eng Technol, vol.19, no.1, pp. 716. Feb. 1995

[6] Roth, B.; Cohen, L.; Hallett, M.; Friauf, W.; Basser, P., "A Theoretical Calculation Of the Electric Field Induced by Magnetic Stimulation of a Peripheral Nerve,"

Muscle \& Nerve, vol.13, no.8, pp. 1097-4598. Aug. 1990

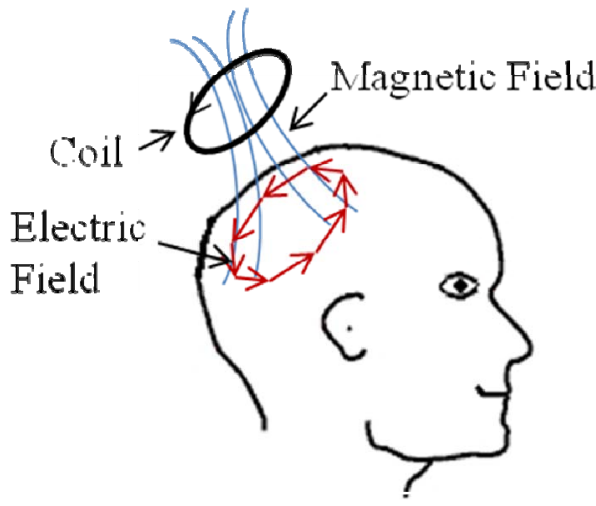

Fig. 1. TMS procedure

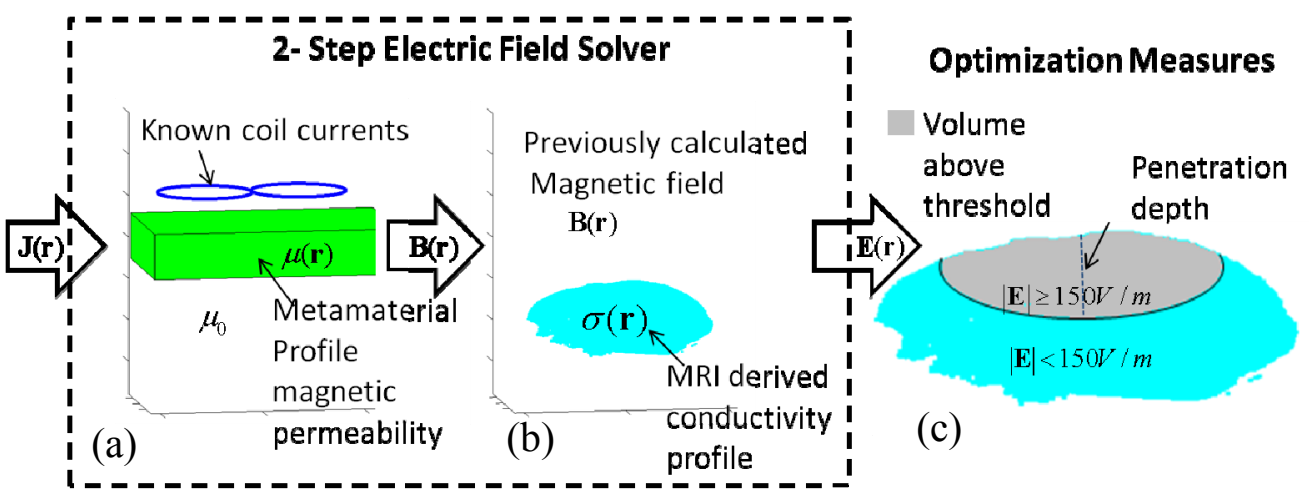

Fig. 2. Schematic of the numerical methods 


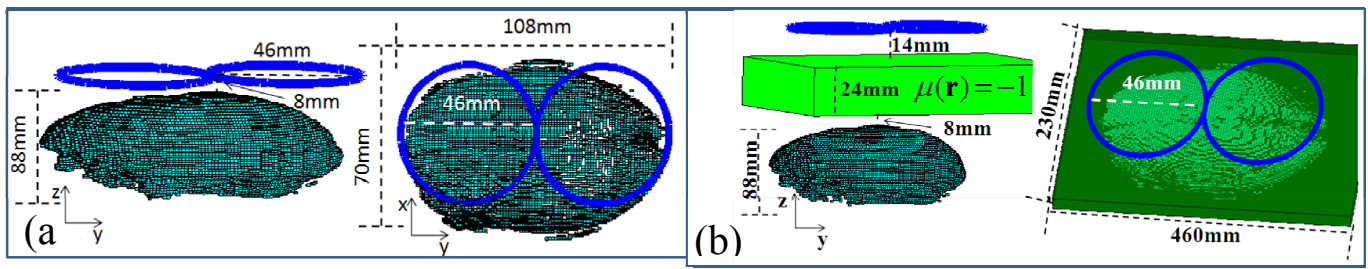

Fig. 3. Simulation scenarios (a) without MM lens (b) with MM lens
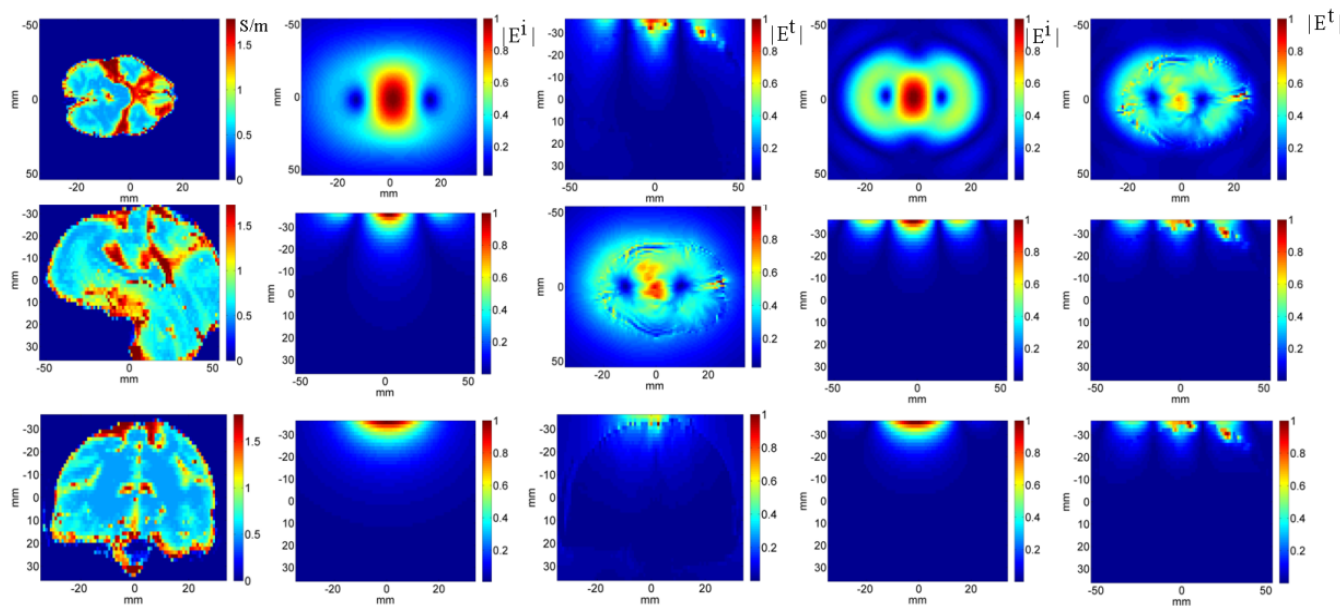

Fig. 4. Row 1: transverse view $6 \mathrm{~mm}$ into the brain; row 2: coronal view through center of the brain; row 3: sagittal view through center of the brain. Col. 1: conductivities for each plane; cols. 2-3: electric field magnitudes for the setup shown in Fig 3a; cols. 4-5: electric field magnitudes for the setup shown in Fig. 3 b.

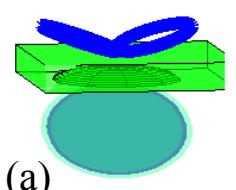

(a)

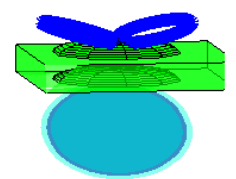

(c)

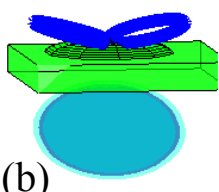

(b)

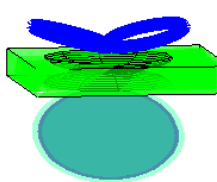

(d)

Fig. 5. (a) flat-concave lens (b) convex-flat lens (c) convex-concave lens (d) concave-concave lens

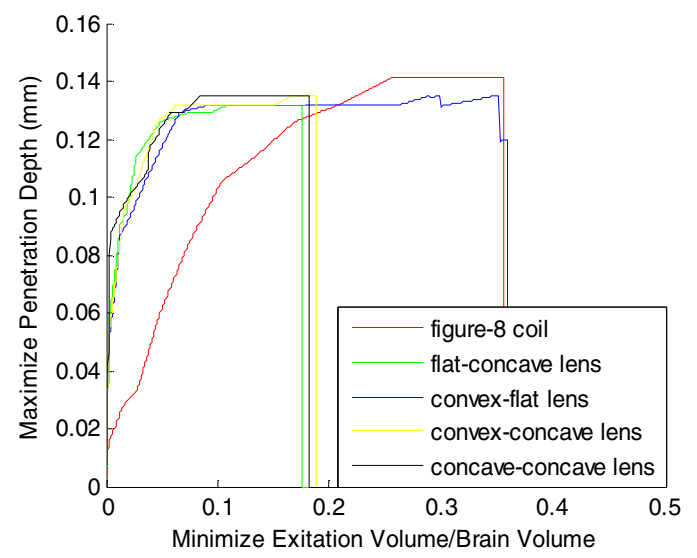

Fig. 6. Pareto fronts derived from analyzing designs shown in Fig. 5. 